\title{
Quantification of dynamic excitation potential of pedestrian population crossing footbridges
}

\author{
Stana Živanovića,* and Aleksandar Pavićb

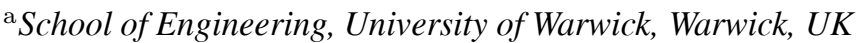 \\ ${ }^{\mathrm{b}}$ Department of Civil and Structural Engineering, University of Sheffield, Sheffield, UK
}

Received 24 June 2009

Revised 6 January 2010

\begin{abstract}
Due to their slenderness, many modern footbridges may vibrate significantly under pedestrian traffic. Consequently, the vibration serviceability of these structures under human-induced dynamic loading is becoming their governing design criterion. Many current vibration serviceability design guidelines, concerned with prediction of the vibration in the vertical direction, estimate a single response level that corresponds to an "average" person crossing the bridge with the step frequency that matches a footbridge natural frequency. However, different pedestrians have different dynamic excitation potential, and therefore could generate significantly different vibration response of the bridge structure. This paper aims to quantify this potential by estimating the range of structural vibrations (in the vertical direction) that could be induced by different individuals and the probability of occurrence of any particular vibration level. This is done by introducing the inter- and intra-subject variability in the walking force modelling. The former term refers to inability of a pedestrian to induce an exactly the same force with each step while the latter refers to different forces (in terms of their magnitude, frequency and crossing speed) induced by different people. Both types of variability are modelled using the appropriate probability density functions. The probability distributions were then implemented into a framework procedure for vibration response prediction under a single person excitation. Instead of a single response value obtained using currently available design guidelines, this new framework yields a range of possible acceleration responses induced by different people and a distribution function for these responses. The acceleration ranges estimated are then compared with experimental data from two real-life footbridges. The substantial differences in the dynamic response induced by different people are obtained in both the numerical and the experimental results presented. These results therefore confirm huge variability in different people's dynamic potential to excite the structure. The proposed approach for quantifying this variability could be used as a sound basis for development of new probability-based vibration serviceability assessment procedures for pedestrian bridges.
\end{abstract}

Keywords: Footbridge, vibration serviceability, probabilistic model, walking, dynamic excitation potential, variability

\section{Introduction}

Due to their slenderness, new footbridges are nowadays more susceptible to vibration serviceability problems under human-induced load than they were in the past [1]. To predict the vibration response of a new structure it is necessary to model accurately both the human-induced dynamic loading and structural dynamic properties. Probably the first codified model of the walking force induced in the vertical direction has been defined in BS 5400 [2] in the 1970s. This model requires calculation of the response to a single "average" person walking across a footbridge at a frequency that matches one of the natural frequencies of the footbridge. The human-induced walking force is modelled as a sinusoidal, and therefore deterministic, force moving across the bridge at a constant speed and having

\footnotetext{
*Corresponding author: Dr. Stana Živanović, University of Warwick, School of Engineering, Coventry CV4 7AL, UK. Tel.: +44 24 765 28392; Fax: +44 24764 18922; E-mail: s.zivanovic@warwick.ac.uk.
} 
predefined constant amplitude. The reason for choosing this resonant force model is that it is considered as the worst-case scenario. This time-domain deterministic approach is widely used worldwide. In recent years, some new and interesting approaches to modelling the walking force induced by a single pedestrian have been developed, but still did not find their way into the design guidelines mainly due to their complexity. Some examples of these are a frequency domain model that takes into account the narrow-band nature of human-induced force [3] and its improvement that relies on Monte Carlo simulations [4]. Additionally, since the infamous problem with excessive lateral vibrations of the London Millennium Bridge in 2000 [5], the research community worldwide has been attracted by this new challenge to study lateral vibration response of footbridges under crowd load [5-8]. However, the design approach to check for vibration serviceability of footbridges in the vertical direction has remained where it was in the 1970s. This is the reason to concentrate on commenting on and evaluating the BS 5400 approach in this paper, devoted exclusively to the vibration response in the vertical direction. The main shortcomings of the BS deterministic model are:

- It does not take into account inter-subject variability, i.e. that different people generate different forces during walking, and therefore have different excitation potential [9].

- It neglects intra-subject variability, i.e. that a pedestrian can never repeat two exactly the same steps [3].

- It assumes that the resonant condition is achieved under a single person walking on an as-built footbridge. However, it is very often difficult to match the footbridge natural frequency during walking, especially when the natural frequency requires either too slow or too fast pacing for an average pedestrian.

- The assessment approach is based on a binary pass-fail philosophy which is more suitable for ultimate than for vibration serviceability limit state.

As a consequence of these shortcomings and simplifications, the existing harmonic force model often significantly overestimates or underestimates experimentally measured footbridge responses. The level of disagreement depends on viability of parameters (forcing amplitude, step length and frequency) used for describing the average person and the walking style of people taking part in the experiments.

To overcome these shortcomings and model reality better, which is the key aim when checking as-built vibration serviceability in day to day operation, it is preferable to consider the whole population of pedestrians using the bridge instead of singling out an "average" person only. To model the forces induced by different people a probabilistic framework for force modelling and response prediction is required. In such an approach, the modelling parameters could be described via their probability density functions and therefore introduced into calculation via their probability of occurrence. The main factors selected to represent the inter-subject variability are: walking (step) frequency, step length and magnitude of the dynamic force [4]. The probability distribution functions for the three parameters are presented in this paper based on currently available data. These distributions could easily be changed depending on characteristics of the pedestrian population of interest. They typically depend on the purpose of the footbridge analysed, its environment, geographic location, etc. For example, it seems that people in Japan generally walk with faster step frequency than people in Montenegro, as will be demonstrated in Section 2.2. This is probably a consequence of the way of life in these two countries as well as the fact that Montenegrins are, on average, taller than Japanese. In addition to the inter-subject variability, the intra-subject variability in the walking-induced force is also modelled via probability density function that quantifies now well known inability of a pedestrian to make exactly the same step twice [3].

It should be mentioned that Ebrahimpour et al. [10] have been working on a probability approach for modelling the walking force. They observed the importance of inter- and intra-subject variability in modelling. However, more than a decade after their work there is still no a single design guideline featuring a probabilistic force model - neither for a single person nor for the crowd loading. A recent work by the authors [4] pursued the probabilistic approach, implementing it for complex multi-mode responses of footbridges. However, this approach based on Monte Carlo simulations might not be the most efficient way to deal with simple footbridges that respond dominantly in a single vibration mode.

This paper concentrates on probabilistic modelling of the vertical component of the walking force induced by a single person on a bridge which vibration response is dominated by a single mode. The aim is to quantify the range of dynamic responses that could be generated by different walkers. This is considered to be a prudent way forward to update the current single person walking model featuring in many design guidelines, such as BS5400 in 
the UK [2] and Ontario Code in Canada [11]. Similarly, the uncertainties in the dynamic parameters (modal mass, damping ratio and natural frequency) describing the structure that vibrates due to the walking excitation could be included into a future framework for probabilistic assessment of human-induced vibration. A model of this kind would be able to estimate the vibration response of the structure in probabilistic sense. Finally, this estimate could then be combined with the probabilistic model of human perception of vibration of the kind suggested in [12] to judge the vibration serviceability of a footbridge against walking excitation. However, probabilistic modelling of both the structure and the human response to vibration are outside the scope of this paper. Instead, the emphasis is on different excitation potential of different people via probabilistic force modelling.

In this paper firstly the main modelling assumptions are defined. Then, a probability based procedure for modal response calculation is explained and applied to two footbridges with experimentally estimated modal properties. Based on this, the probability of having a certain level of vibration is obtained. The range of calculated vibration response levels was compared with the single value from a deterministic procedure and checked against some available real-life measurements.

\section{Modelling assumptions}

This section aims to describe the parameters important for the probabilistic modelling of the walking force. These are: walking frequency, force amplitude, step length and imperfections in human walking. Probability distributions of these random variables are suggested based either on the data available in literature or the data gathered previously by the authors [1,13]. Based on experience from full scale lively footbridges where the first harmonic of the walking force is often responsible for generating strong structural vibrations [1], it was decided to consider only this harmonic in this paper.

\subsection{Footbridge as SDOF system}

Footbridges are structures that often have well separated frequencies of vibration modes, each of which could be modelled as a single degree of freedom (SDOF) system with known modal properties (natural frequency, modal mass and damping ratio). Among these modes, usually only one is responsible for the footbridge liveliness [14]. The shape of this mode could often be approximated by a half-sine function. These assumptions are used in the study presented.

\subsection{Walking (step) frequency}

Matsumoto et al. [15] identified the normal distribution of human walking frequencies with the mean value of $2.00 \mathrm{~Hz}$ and standard deviation of $0.173 \mathrm{~Hz}$. This was identified using a sample of 505 people. Recently, a more extensive work was conducted on a full scale pedestrian bridge in Montenegro where the step frequency was estimated by analysing video records of 1976 people during their crossing over the footbridge [13]. It was confirmed that the distribution of human walking frequencies follows the normal distribution, but with mean value of $1.87 \mathrm{~Hz}$ and standard deviation of $0.186 \mathrm{~Hz}$ (Fig. 1a). As already mentioned, it is likely that the distribution parameters differ between different countries (say, between Japan and Montenegro), different footbridge locations and utilisations, etc. In this paper, the distribution identified on the footbridge in Montenegro is used since this footbridge is one of the two structures investigated and presented here. Also, this distribution has the mean value that is closer to that found by some other European researchers in recent years: $1.9 \mathrm{~Hz}$ reported by Kerr \& Bishop [16] and $1.8 \mathrm{~Hz}$ by Pachi \& Ji [17], as well as by Sahnaci \& Kasperski [18].

To incorporate the probability density function shown in Fig. 1a into the calculation of footbridge response, it is convenient to transform the horizontal axis into a frequency ratio between the step frequency and the natural frequency of a particular footbridge. An example for a footbridge with natural frequency of $2.04 \mathrm{~Hz}$ is presented in Fig. 1b. It can be seen that frequency ratio ranges between 0.64 and 1.19 for this particular bridge. During the transformation the vertical axis was multiplied by $2.04 \mathrm{~Hz}$ to preserve the area defined by the probability density function being dimensionless number equal to 1 [19]. 

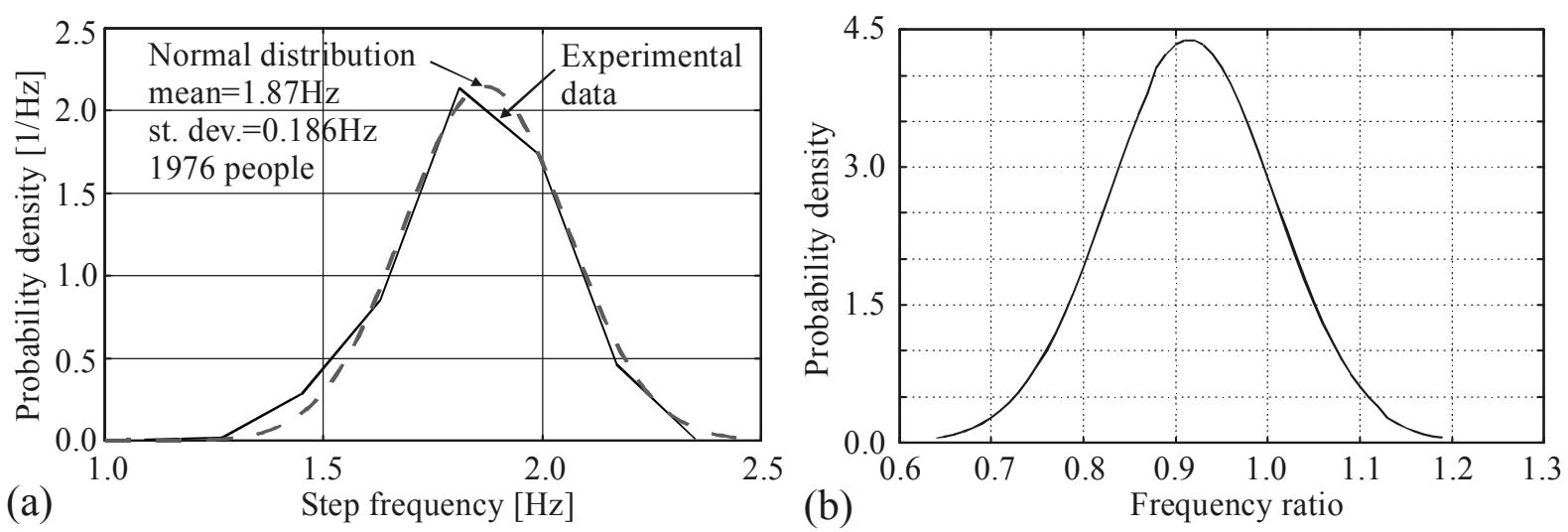

Fig. 1. (a) Normal distribution of walking frequencies. (b) Normal distribution when the frequency axis is normalised to a footbridge natural frequency in the vertical direction $(2.04 \mathrm{~Hz}$ in this example).
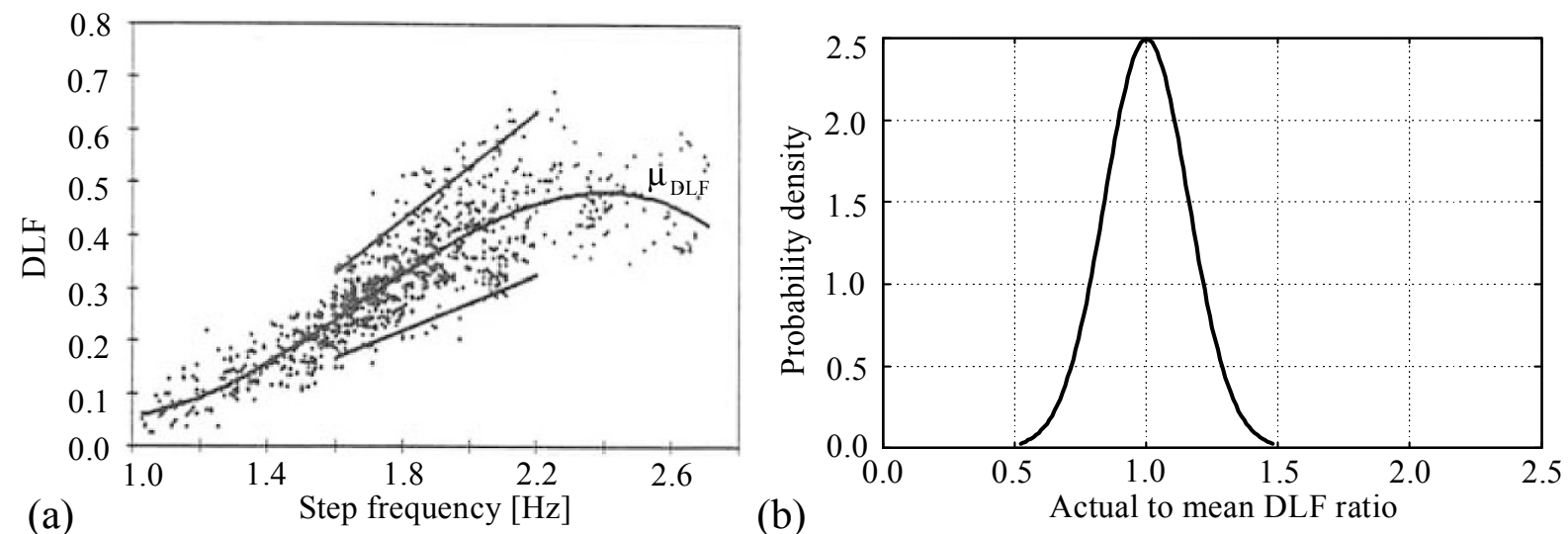

Fig. 2. (a) DLF for first harmonic of the walking force (after Kerr, 1998). (b) Distribution of actual to mean DLF ratio for the first harmonic of the force induced by walking.

\subsection{Step length}

The length of a step made during walking differs between different individuals [17]. The step length $l_{s}$ multiplied by the step frequency $f_{s}$ equals to the walking speed $v_{p}$, which determines the time a pedestrian needs for crossing a walking path specified. More attention to collecting the experimental data about the relationship between the walking parameters on as-built footbridges has been paid in recent years [17,20]. For example, it was found that the degree of linear correlation between the walking frequency and step length is very small [20]. This suggests that these two parameters could be treated as independent random variables. Similarly to the step frequency, the step length can also be modelled as normally distributed with mean value of $0.71 \mathrm{~m}$ and standard deviation of $0.071 \mathrm{~m}[17,20]$.

\subsection{Force amplitude}

When modelling the walking-induced force as a harmonic force, its amplitude is usually defined as a portion of the pedestrian's weight, that is as a product of a dimensionless coefficient called Dynamic Load Factor (DLF) and the pedestrian's weight $W$. The most extensive research to date into DLFs was conducted by Kerr [9]. He analysed about 1000 force records produced by 40 test subjects and presented DLFs for different force harmonics as a function of the walking frequency. DLFs for the first harmonic as obtained by Kerr are shown in Fig. 2a. The dependence of the approximate mean value of DLF $\mu_{\mathrm{DLF}}$ on the step frequency $f_{s}$ is given by: 


$$
\mu_{\mathrm{DLF}}=-0.2649 f_{s}^{3}+1.3206 f_{s}^{2}-1.7597 f_{s}+0.7613
$$

Kerr [9] also found that in the normal walking frequency range 1.5-2.2 Hz, 95\% of DLFs lie in the area $\mu_{\mathrm{DLF}} \pm$ $0.32 \mu_{\mathrm{DLF}}$. Under an assumption that DLFs are normally distributed around their mean value (for a specific walking frequency), the standard deviation can be defined as $\sigma_{\mathrm{DLF}}=0.16 \mu_{\mathrm{DLF}}$.

The described probability density function for a DLF can be normalised to the value of the mean DLF (Fig. 2b). It should be noticed that this function does not depend on the walking frequency. Since the modal response of a linear SDOF footbridge model is directly proportional to the DLF then, for example, 1.5 times increase in the DLF will generate 1.5 times higher modal response. By this analogy it can be concluded that the probability of the ratio between the actual modal acceleration response and the response to the mean value of DLF is the same as the probability of the ratio between the actual DLF and the mean DLF. This enables the use of only the mean DLF when calculating structural modal response. After this calculation the probability that this 'mean' response is different from the actual one can be estimated.

Regarding pedestrian weight $W$ there are indications that its increase leads to increase of DLFs [21]. However, an explicit quantification of this dependence is yet to be made. Also, it is difficult to find a precise description of the probability distribution of the pedestrian weight (i.e. its type and parameters describing it), although some (incomplete) information is available [22]. These were the reasons to omit this distribution from the analysis and use an average weight of $750 \mathrm{~N} \mathrm{[22]} \mathrm{in} \mathrm{the} \mathrm{formulation} \mathrm{of} \mathrm{the} \mathrm{force} \mathrm{model.} \mathrm{When} \mathrm{more} \mathrm{data} \mathrm{describing} \mathrm{the} \mathrm{distribution}$ of the weight and its relationship with the DLFs are collected, they can easily be combined with the probability distribution of DLFs to a single probability distribution defining the amplitude of the sinusoidal walking force $(\mathrm{DLF} \cdot \mathrm{W})$.

\subsection{Intra-subject variability in force}

So far in this paper the inter-subject variability in the walking force was modelled via appropriate probability distributions related to the step frequency, the step length and the force amplitude. The two parameters: step frequency $f_{s}$ and force magnitude DLF . W fully describe a harmonic force model, while the step length $l_{s}$, multiplied by step frequency, defines the walking speed which in turn determines the time spent on crossing the bridge. Therefore, so far the walking force was assumed to be periodic so that its first harmonic could be modelled as a sinusoidal force. Under this force the modal acceleration response $a_{\sin }(t)$ can be calculated, taking into account the half-sine mode shape. However, in the same way that some people are able to walk steadily and induce an almost perfectly sinusoidal first harmonic [23], there are many more people who cannot do this. Due to intra-subject variability the force induced in every step is usually slightly different in terms of its frequency content and amplitude and it should be treated as a narrow band random process rather than a sinusoidal force $[3,18]$

One way to consider this, and at the same time to keep simplicity of the response calculation corresponding to harmonic force excitation, is to define the probability that the modal response to an actual (measured) walking force $a_{\mathrm{c}}(t)$ will be different from that generated by a sine force $a_{\mathrm{sin}}(t)$. Examples of such responses are shown in Fig. 3 as black and grey lines, respectively. The corresponding peak responses in Fig. 3 are denoted as $A_{c}$ and $A_{s i n}$.

Figure 3 a presents the two modal responses when a pedestrian walks at a step frequency that matches a footbridge natural frequency, while in Fig. $3 \mathrm{~b}$ they walk at a step frequency equal to $80 \%$ of the natural frequency. In the latter case, the beating response, noticed in some real-life measurements when walking with out-of-resonance frequency, is present in the calculated response to the treadmill-measured walking time history (Fig. 3b black-dashed line). This pattern is almost non-existent in the case of simulation due to the sinusoidal force (Fig. $3 \mathrm{~b}$ grey line).

The ratio between the two peak modal responses will be called the correction coefficient $c$ :

$$
c=\frac{A_{\mathrm{c}}}{A_{\mathrm{sin}}}
$$

and it is this ratio factor that will be used for introducing the intra-subject variability into calculation of the actual peak modal response.

To define the probability density function for the correction coefficient $c, 95$ walking forces measured by Brownjohn et al. [3] on a treadmill were analysed. These walking forces were produced by eight test subjects who were asked to walk for at least $60 \mathrm{~s}$ on a treadmill set to a constant speed. The speed range was between $2.5 \mathrm{~km} / \mathrm{h}$ and $8.0 \mathrm{~km} / \mathrm{h}$ 

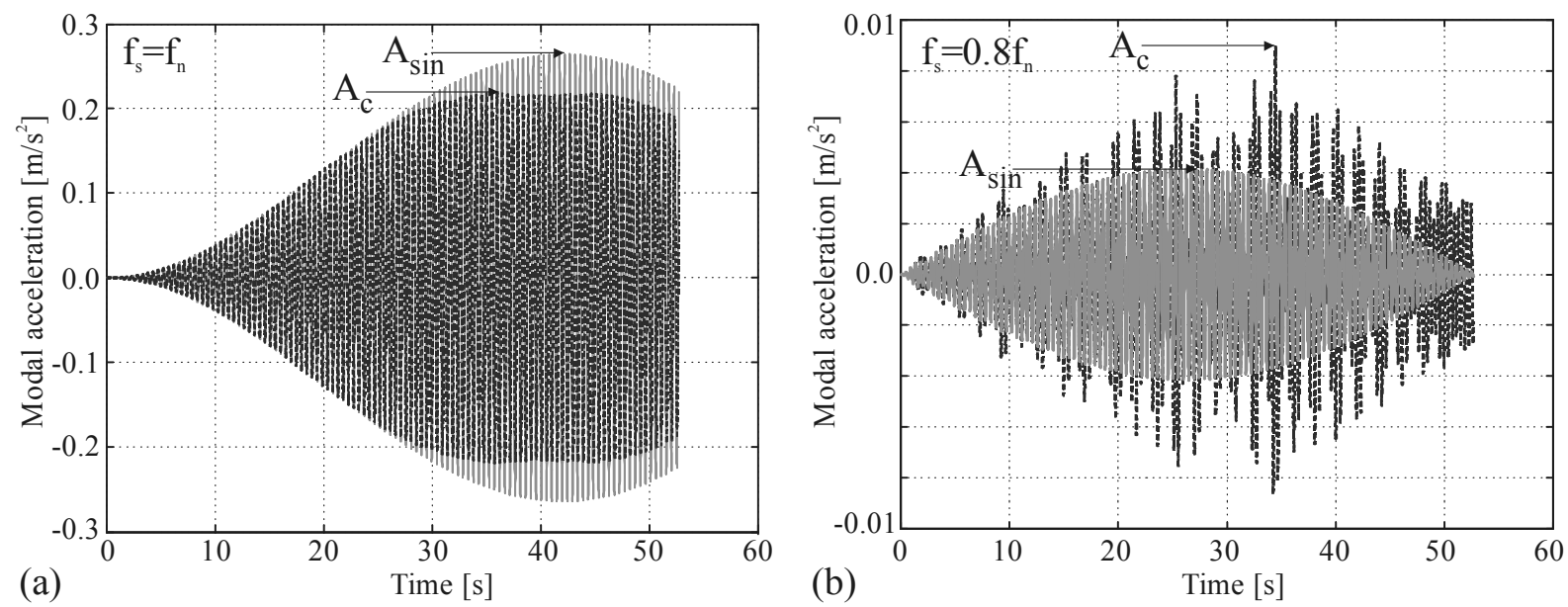

Fig. 3. Comparison of modal responses due to measured force (black-dashed line) and corresponding sine force (grey line) when walking at (a) resonant frequency and (b) non-resonant frequency.

in different tests. Therefore, the walking speed was set to a constant value during each test, while the walking frequency was freely chosen by each test subject so that they could walk in a comfortable manner.

The measured walking forces were band-pass filtered around the walking frequency so that only force components pertinent to the first harmonic remained. Then, the filtered force was multiplied by the half sine mode shape to get the modal force. Finally, peak modal acceleration response of the SDOF model to this force was calculated. The natural frequency of the footbridge (i.e. the SDOF system) was studied parametrically and assumed to be in the range between $f_{s} / 1.20$ and $f_{s} / 0.80$, where $f_{s}$ is the average walking frequency, while modal damping ratio ranged between $0.1 \%$ and $2.0 \%$. For each response calculation the peak modal acceleration response $A_{\mathrm{c}}$ obtained in this way was divided by the peak modal acceleration response $A_{\text {sin }}$ due to a corresponding sinusoidal force to calculate the correction coefficient $c$. The amplitude of the sinusoidal force was defined as the average amplitude of the filtered force measured on the treadmill.

A general observation from the results obtained is that the peak modal acceleration due to measured walking force is attenuated when walking in resonance in comparison with that produced by the corresponding sinusoidal force, i.e. the correction coefficient is less than 1 (Fig. 3a). This is a consequence of the inability of the test subject to walk at the constant frequency all the time. It should, however, be noticed that some test subjects produced almost perfect sine force leading to the correction coefficient equal to 1 . In few cases, the correction coefficient was even greater than 1. This was a consequence of having several heavy footfalls (i.e. footfalls in which the force amplitude was higher than the average one) occurring when vibrations have already been well developed. On the other hand, when the step frequency is away from the natural frequency, the correction coefficient is mainly greater than 1 (Fig. 3b). The reason for this are again imperfections in human walking frequency from one step to another, but this time its slight change leads to being closer to the resonant frequency from time to time, causing an actual acceleration response to be greater than the one generated by a sine force.

Different distributions for fitting the correction coefficient data (for specific combination of damping ratios and natural frequencies) were tried. Among them, it was found that a gamma distribution best described the probability distribution of the correction coefficient. An example of the quality of the approximation can be seen in Fig. 4.

The gamma distribution could describe a trend that with increasing damping ratio the scatter of the calculated correction coefficient decreases, with the most probable correction coefficient approaching 1.0. This makes sense considering that the sharpness of the resonant peak in the frequency domain decreases with increasing damping. An example of resulting gamma distributions for $f_{s}=1.15 f_{n}$ is shown in 5a. Also, the gamma distribution can represent the fact that for non-resonant walking, the increase in the walking to natural frequency ratio leads to smaller scatter in the correction coefficient, with its peak approaching the value equal to 1 . An example for a bridge with a damping ratio of $\zeta=0.4 \%$ is presented in Fig. 5 b.

The probability density function $f(x)$ for the gamma distribution is defined by the following formula [19]: 


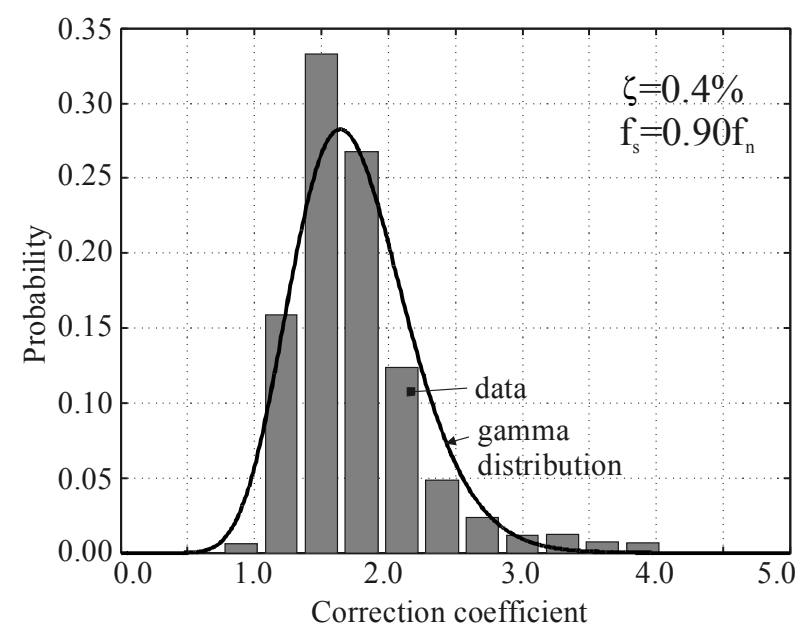

Fig. 4. Distribution of the correction coefficient when walking at pacing rate equal to $0.9 f_{n}$.
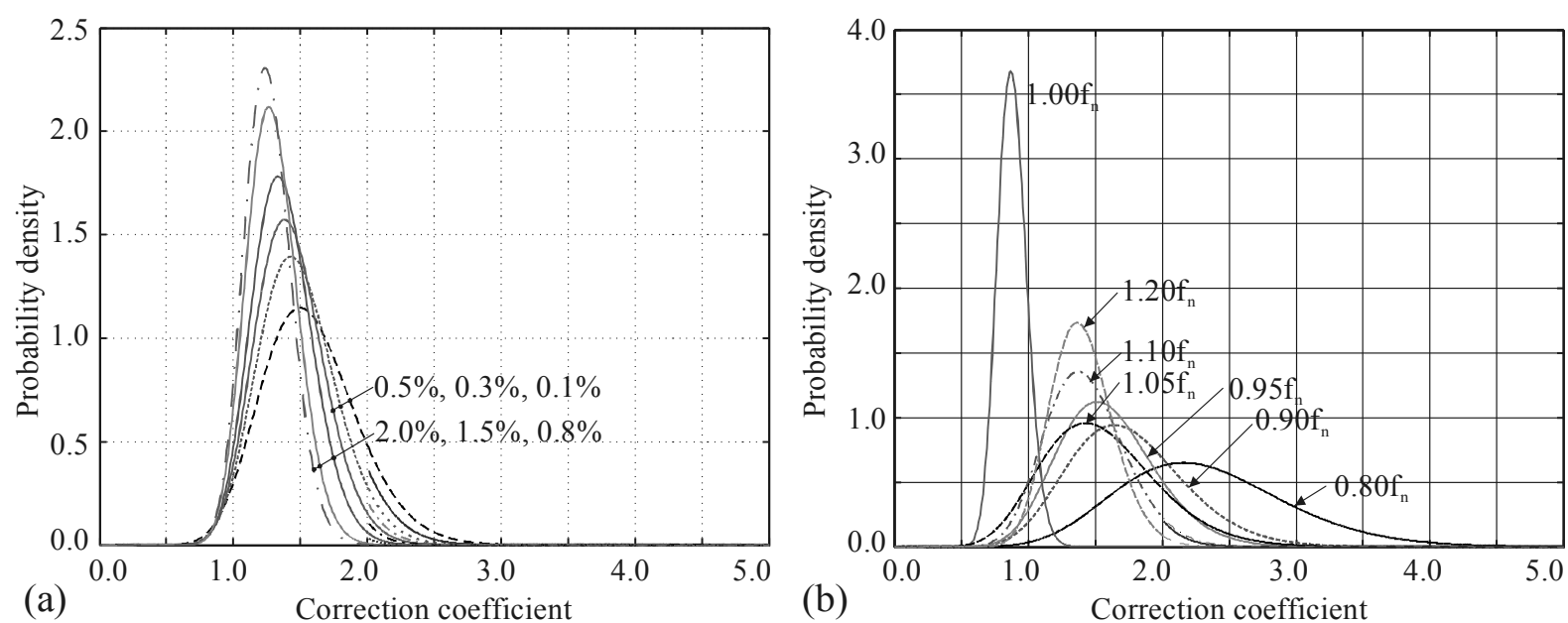

Fig. 5. (a) Gamma distributions for the correction coefficient when $f_{s}=1.15 f_{n}$ for footbridges with different damping. (b) Gamma distributions for the correction coefficient when walking at different frequencies $f_{s}$ on a bridge with damping ratio equal to $0.4 \%$.

$$
f(x)=\frac{x^{a-1} e^{-\frac{x}{b}}}{b^{a} \int_{0}^{\infty} x^{a-1} e^{-x} d x}
$$

where $x$ is the random variable (i.e. correction coefficient $c$ ). The distribution shape is uniquely determined by parameters $a$ and $b$. The parameters were identified for footbridges with different damping ratios and different walking frequencies (Table 1 and Table 2). For a footbridge with an arbitrary damping ratio and/or frequency ratio the two parameters could be approximated using closest values of damping and frequency ratios available in the tables.

\section{Prediction of footbridge response to single person walking}

This section describes the general framework for probabilistic description of footbridge vibration response to a single person crossing. The framework methodology is described step-by-step so that it can be implemented on 
Table 1

Parameter $a$ of gamma distributions

\begin{tabular}{rcccccccccc}
\hline & \multicolumn{10}{c}{ Damping ratio [\%] } \\
\cline { 2 - 10 }$f_{s} / f_{n}$ & 0.1 & 0.2 & 0.3 & 0.4 & 0.5 & 0.6 & 0.8 & 1.0 & 1.5 & 2.0 \\
\hline 0.80 & 12.227 & 12.622 & 13.001 & 13.587 & 14.454 & 15.166 & 16.470 & 17.353 & 19.560 & 22.013 \\
0.85 & 12.646 & 13.735 & 14.510 & 16.177 & 17.706 & 19.067 & 21.345 & 23.200 & 26.629 & 29.289 \\
0.90 & 10.353 & 12.251 & 14.350 & 16.197 & 17.846 & 19.273 & 21.543 & 23.444 & 27.443 & 31.284 \\
0.95 & 13.967 & 16.214 & 17.874 & 19.251 & 20.478 & 20.701 & 20.170 & 22.874 & 30.449 & 39.159 \\
1.00 & 31.431 & 41.659 & 52.926 & 65.341 & 78.930 & 93.821 & 126.460 & 161.180 & 247.620 & 318.600 \\
1.05 & 10.061 & 10.886 & 11.934 & 12.934 & 13.744 & 14.415 & 13.112 & 15.665 & 23.409 & 33.318 \\
1.10 & 18.589 & 20.680 & 21.812 & 22.868 & 23.721 & 24.045 & 24.268 & 22.713 & 28.083 & 34.101 \\
1.15 & 19.687 & 23.154 & 26.071 & 28.565 & 30.816 & 32.872 & 36.517 & 39.651 & 46.277 & 52.192 \\
1.20 & 24.319 & 28.624 & 32.605 & 36.314 & 39.736 & 42.967 & 48.834 & 53.939 & 64.153 & 71.769 \\
\hline
\end{tabular}

Table 2

Parameter $b$ of gamma distributions

\begin{tabular}{ccccccccccc}
\hline & \multicolumn{10}{c}{ Damping ratio [\%] } \\
\cline { 2 - 11 }$f_{s} / f_{n}$ & 0.1 & 0.2 & 0.3 & 0.4 & 0.5 & 0.6 & 0.8 & 1.0 & 1.5 & 2.0 \\
\hline 0.80 & 0.2202 & 0.2019 & 0.1870 & 0.1715 & 0.1550 & 0.1429 & 0.1246 & 0.1133 & 0.0928 & 0.0777 \\
0.85 & 0.1795 & 0.1567 & 0.1422 & 0.1226 & 0.1084 & 0.0979 & 0.0836 & 0.0743 & 0.0609 & 0.0530 \\
0.90 & 0.1922 & 0.1541 & 0.1260 & 0.1080 & 0.0954 & 0.0863 & 0.0745 & 0.0665 & 0.0539 & 0.0455 \\
0.95 & 0.1250 & 0.1038 & 0.0915 & 0.0829 & 0.0762 & 0.0741 & 0.0740 & 0.0632 & 0.0447 & 0.0332 \\
1.00 & 0.0261 & 0.0203 & 0.0163 & 0.0135 & 0.0113 & 0.0097 & 0.0073 & 0.0059 & 0.0039 & 0.0031 \\
1.05 & 0.1673 & 0.1500 & 0.1330 & 0.1197 & 0.1103 & 0.1032 & 0.1114 & 0.0900 & 0.0563 & 0.0376 \\
1.10 & 0.0820 & 0.0719 & 0.0668 & 0.0627 & 0.0596 & 0.0581 & 0.0565 & 0.0596 & 0.0464 & 0.0371 \\
1.15 & 0.0801 & 0.0659 & 0.0570 & 0.0509 & 0.0463 & 0.0427 & 0.0375 & 0.0338 & 0.0279 & 0.0241 \\
1.20 & 0.0618 & 0.0510 & 0.0438 & 0.0386 & 0.0348 & 0.0317 & 0.0273 & 0.0243 & 0.0198 & 0.0173 \\
\hline
\end{tabular}

(a)

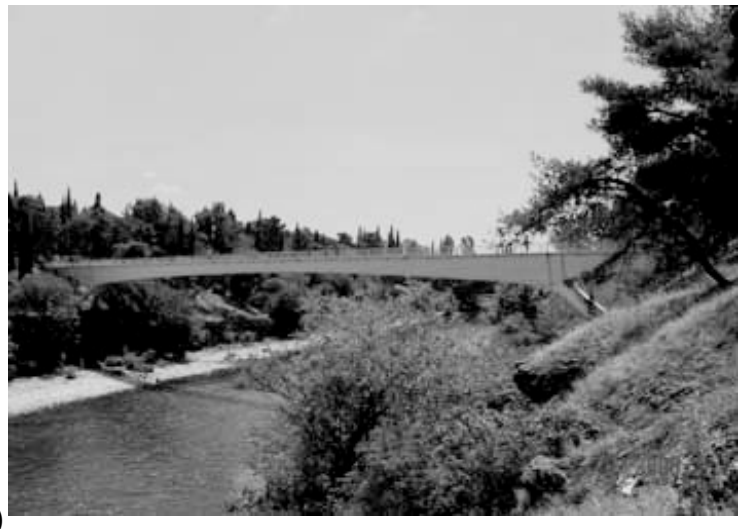

(b)

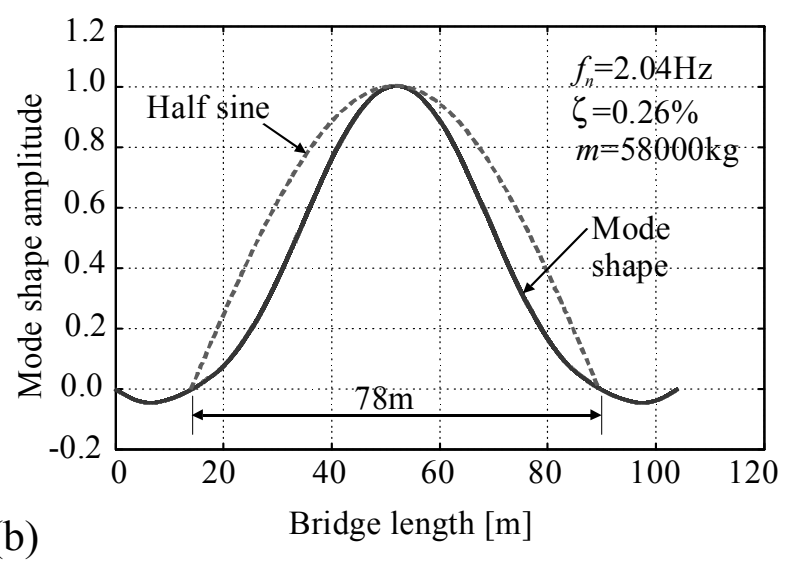

Bridge length $[\mathrm{m}]$

Fig. 6. Footbridge 1 - (a) photograph and (b) modal properties of the fundamental mode of vibration.

generic footbridge structures where a vertical dynamic excitation due to a single person walking is a relevant design criterion. Statistical distributions for different parameters defined in the previous section are used for this purpose. The procedure is applied on two pilot structures. First, an as-built footbridge in Montenegro where only the first walking harmonic was relevant and the mode shape could be approximated by a half sine function is investigated. After this, the procedure is repeated for another footbridge.

The footbridge in Montenegro (hereafter referred to as Footbridge 1) is a steel box girder footbridge shown in Fig. 6a. Its length is $104 \mathrm{~m}$, with $78 \mathrm{~m}$ between inclined columns. The footbridge responds to normal walking excitation dominantly in the first vibration mode with frequency at $2.04 \mathrm{~Hz}$ [13]. The mode shape and modal properties of this mode (natural frequency $f_{n}$, damping ratio $\zeta$ and modal mass $m$ ) as measured are shown in Fig. $6 \mathrm{~b}$. 

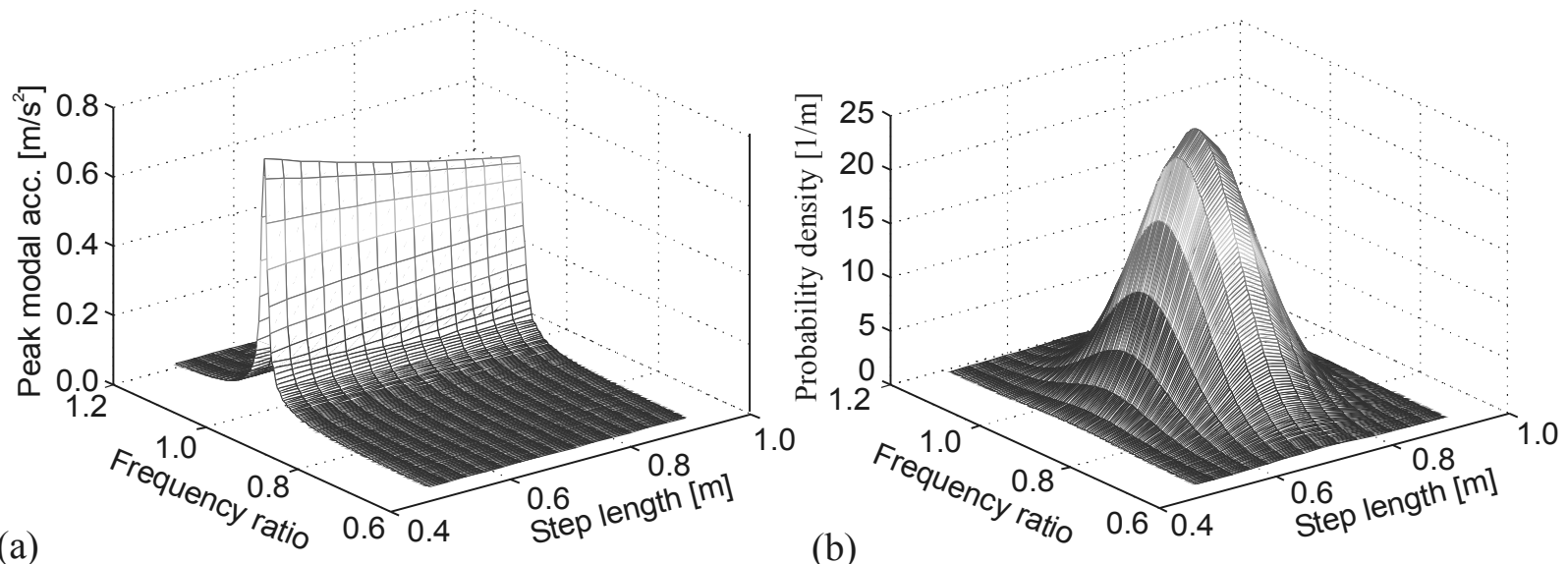

(b)

Fig. 7. (a) Peak modal acceleration response due to sinusoidal walking force and (b) joint probability density function for different combinations of step frequency and step length during the footbridge crossing.

\subsection{Peak modal response to sinusoidal excitation}

The first step in the analysis is to calculate the peak modal response of the SDOF system to sinusoidal excitation. Assuming the half-sine mode shape, the equation of motion in its modal form can be written as [24]:

$$
a(t)+2 \zeta\left(2 \pi f_{n}\right) v(t)+\left(2 \pi f_{n}\right)^{2} d(t)=\frac{1}{m} \underbrace{D L F \cdot W \cdot \sin \left(2 \pi f_{s} t\right)}_{F_{\sin }(t)} \cdot \underbrace{\sin \left(\frac{\pi f_{s} l_{s}}{L} t\right)}_{\phi(t)}
$$

where $a(t), v(t)$ and $d(t)$ are modal acceleration, velocity and displacement of the footbridge structure, respectively, while $\zeta, m$ and $f_{n}$ are modal damping, mass and natural frequency, respectively. The right hand side of the equation represents the modal force acting on the SDOF system obtained by multiplication of the sinusoidal force $F_{\text {sin }}(t)$ by the half-sine mode shape $\phi(t)$. The frequency of the force $F_{\sin }(t)$ is $f_{s}$ while its amplitude is defined as a product of the mean DLF dependent on $f_{s}$ (defined in Equation (1)) and an assumed average pedestrian weight $W=$ $750 \mathrm{~N}$ [22]. The mode shape $\phi(t)$ was initially a space function dependent on pedestrian position on the bridge $x$ and footbridge length $L$. However, by assuming a constant pedestrian velocity $v_{p}$, the mode shape defined along the bridge length can be transformed into a time-varying function:

$$
\sin \frac{\pi x}{L}=\sin \frac{\pi v_{p} t}{L}=\sin \left(\frac{\pi\left(f_{s} l_{s}\right)}{L} t\right)
$$

where $l_{s}$ is the step length.

Using simple trigonometric transformations Equation (4) could be rewritten in the form:

$$
a(t)+2 \zeta\left(2 \pi f_{n}\right) v(t)+\left(2 \pi f_{n}\right)^{2} d(t)=\frac{D L F \cdot W}{2 m}\left(\cos \left(2 \pi f_{s}-\frac{\pi f_{s} l_{s}}{L}\right) t-\cos \left(2 \pi f_{s}+\frac{\pi f_{s} l_{s}}{L}\right) t\right)(6)
$$

for which analytical solutions to two cosine modal force terms on the right hand side could be found in literature [24]. Summing up these two solutions the total response of the structure to a harmonic force moving over a bridge characterised by a half-sine mode shape could be determined.

For Footbridge 1 the solution was found for different combinations of step to natural frequency ratios and step lengths (belonging to the previously defined normal distributions). The resulting peak modal acceleration shown in Fig. 7a gives a range of possible peak modal acceleration responses $A_{\text {sin }}$ under sinusoidal force excitation. 


\subsection{Joint probability for walking parameters}

As explained previously, the walking frequency and the step length are independent normally distributed variables. Therefore, the joint probability of walking at a particular frequency $f_{s}$ and having a particular step length $l_{s}$ during a footbridge crossing can be calculated by multiplying the two normal probability density functions [19]. The resulting joint probability density function is shown in Fig. $7 \mathrm{~b}$.

\subsection{Modification of peak modal response $A_{\sin }$ due to intra-subject variability}

For every pair of frequency ratio $f_{s} / f_{n}$ and step length $l_{s}$, it is possible to find the peak modal acceleration $A_{\text {sin }}$ due to a sine force (Fig. 7a) as well as a point in the probability density function $p\left(f_{s} / f_{n}, l_{s}\right)$ (Fig. 7b) that represents exactly this combination of $f_{s} / f_{n}$ and $l_{s}$. After this, the fact that the peak acceleration level $A_{c}$ could be higher or lower than that in Fig. 7a due to intra-subject variability can be introduced. For this purpose the peak acceleration $A_{\text {sin }}$ from Fig. 7a for each point $\left(f_{s} / f_{n}, l_{s}\right)$ is multiplied by the correction coefficient $c$ from the appropriate gamma distribution defined in Section 2.5: $A_{c}=c \cdot A_{\text {sin }}$. In this way a range of possible peak acceleration values $A_{c}$ for each $A_{\text {sin }}$ from Fig. 7a has been obtained.

It should be noted here that the gamma distribution of the correction coefficient chosen for this calculation depends on the walking to natural frequency ratio $\left(f_{s} / f_{n}\right)$. At the same time the probability density function of the three variables $p\left(f_{s} / f_{n}, l_{s}, c\right)$ corresponding to each combination of $f_{s} / f_{n}, l_{s}$ and $c$ used for calculation of $A_{c}$ can be obtained by multiplication of every point in the joint probability density function $p\left(f_{s} / f_{n}, l_{s}\right)$ (Fig. 7b) by gamma probability density function $p(c)$ of the kind presented in Fig. 5:

$$
p\left(f_{s} / f_{n}, l_{s}, c\right)=p\left(f_{s} / f_{n}, l_{s}\right) \cdot p(c) .
$$

As the next step, the probability $P_{c}$ of having peak acceleration $A_{c}$ can be found as:

$$
P_{c}=p\left(f_{s} / f_{n}, l_{s}, c\right) \cdot \Delta\left(f_{s} / f_{n}\right) \cdot \Delta l_{s} \cdot \Delta c
$$

where $\Delta\left(f_{s} / f_{n}\right), \Delta l_{s}$ and $\Delta c$ are discrete steps used in analysis for the variables $f_{s} / f_{n}, l_{s}$ and $c$, respectively.

Finally, the probability $P_{A_{c, i}: A_{c, i+1}}$ that the peak acceleration $A_{c}$ is within a certain interval, such as $A_{c, i} \leqslant A_{c}<$ $A_{c, i+1}$, can be found in the well-known way as follows:

$$
P_{A_{c, i}: A_{c, i+1}}=\sum_{A_{c, i} \leqslant A_{c}<A_{c, i+1}} P_{c} .
$$

\subsection{Influence of DLF variability on peak modal response}

As the final step in the analysis, the influence of probability of DLF being different from the mean value assumed throughout the previous calculation should be taken into account. This can be done by multiplying the probability of the acceleration levels obtained in the previous section with the probability function related to DLF variation (Fig. 2b). After this, the probability that the vibration level is within a certain range can be obtained as presented in Fig. 8a. It can be concluded that excitation potential of different pedestrians might be significantly different. Two extremes in Fig. 8a are that some people generate low acceleration level below $0.05 \mathrm{~m} / \mathrm{s}^{2}$, while some could induce vibration as high as $0.9 \mathrm{~m} / \mathrm{s}^{2}$. However, it is clear from Fig. 8a that as many as $57 \%$ of people belong to the first group while a negligible number belongs to the second group.

A more interesting cumulative probability that the acceleration level is either smaller than or equal to a certain level is presented in Fig. 8b. Having this distribution and assuming that, for example, the acceleration level of $0.35 \mathrm{~m} / \mathrm{s}^{2}$ is unacceptable, the probability of exceedance of this level could be estimated. It can be seen in Fig. $8 \mathrm{~b}$ that this level of vibration is exceeded once in every 20 crossings by a single person (5\% exceedance probability). Naturally, the probability of exceedance of any other vibration level could be obtained from the same figure. 

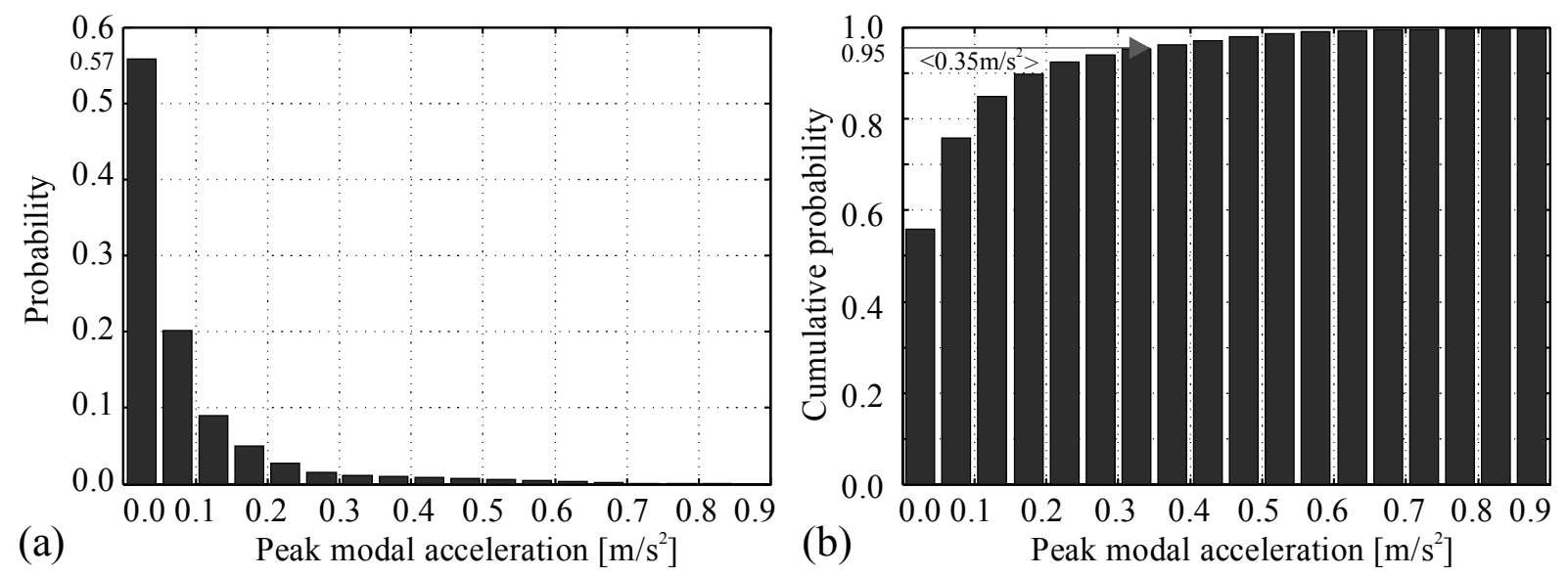

Fig. 8. (a) Final probability of a peak modal acceleration level due to single person crossing the Footbridge 1. (b) Cumulative probability that the acceleration level is smaller than or equal to the acceleration level considered (shown on the horizontal axis).

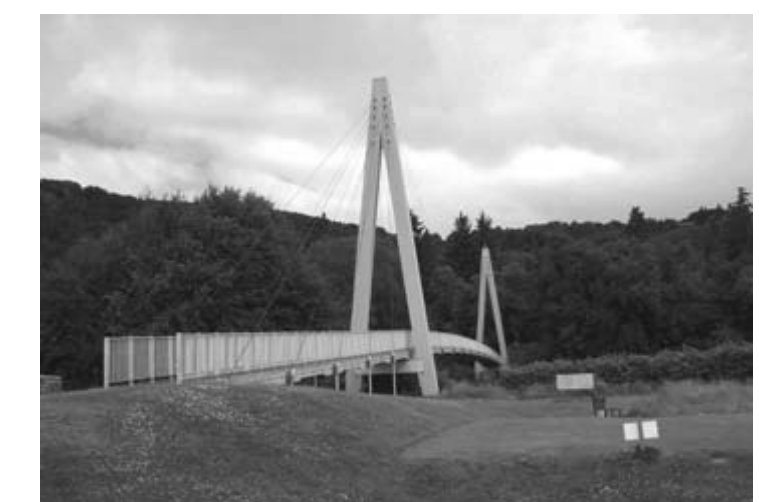

(a)

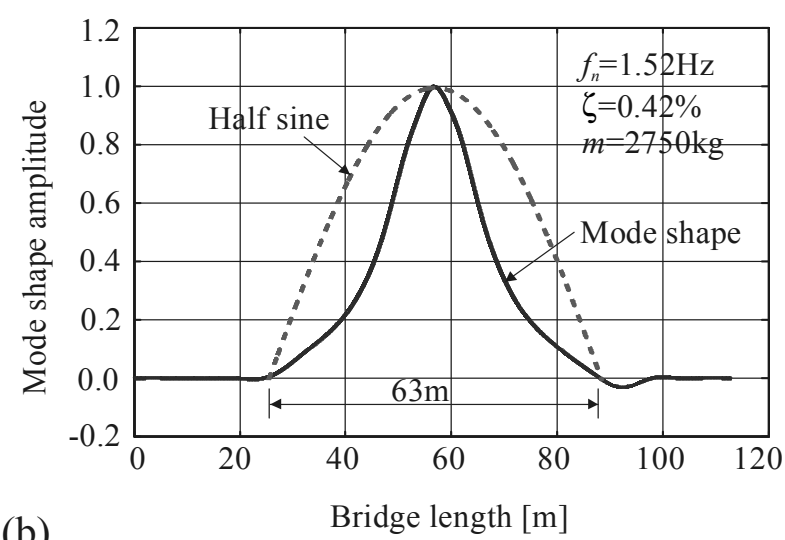

(b)

Fig. 9. Footbridge 2 - (a) photograph and (b) modal properties of the fundamental mode of vibration.

\subsection{Footbridge 2}

The procedure applied to Footbridge 1 is repeated for a light cable-stayed footbridge made of glass reinforced plastic (Footbridge 2). The total length of this footbridge is $113 \mathrm{~m}$ and its total mass is only about $20000 \mathrm{~kg}$. The footbridge is shown in Fig. 9a, and the properties of its first mode of vibration, as measured during modal testing, are given in Fig. 9b.

Footbridge 2 is a very light structure that is prone to significant vibrations generated by human walking. Following exactly the same procedure as for Footbridge 1, the probability distribution of the acceleration response due to excitation of the fundamental vibration mode could be calculated (Fig. 10a). The cumulative distribution of this response is presented in Fig. 10b. From this figure it can be seen that, for example, only $46 \%$ of people (i.e. approximately every second person) would cross the bridge without generating vibrations above $0.35 \mathrm{~m} / \mathrm{s}^{2}$. Also, due to the lightness of the footbridge, nobody can walk and generate vibrations below $0.1 \mathrm{~m} / \mathrm{s}^{2}$.

The result obtained for Footbridge 2 is verified by the response measurements to single person excitation. Seven test subjects were asked to cross the bridge with their 'fast', 'normal' and 'slow' pacing rates. For each pacing rate two tests were conducted. Therefore, in total 42 crossings of the bridge were analysed, and peak modal acceleration was extracted from each of them. In this testing programme, the footbridge response was measured at the midspan point. 

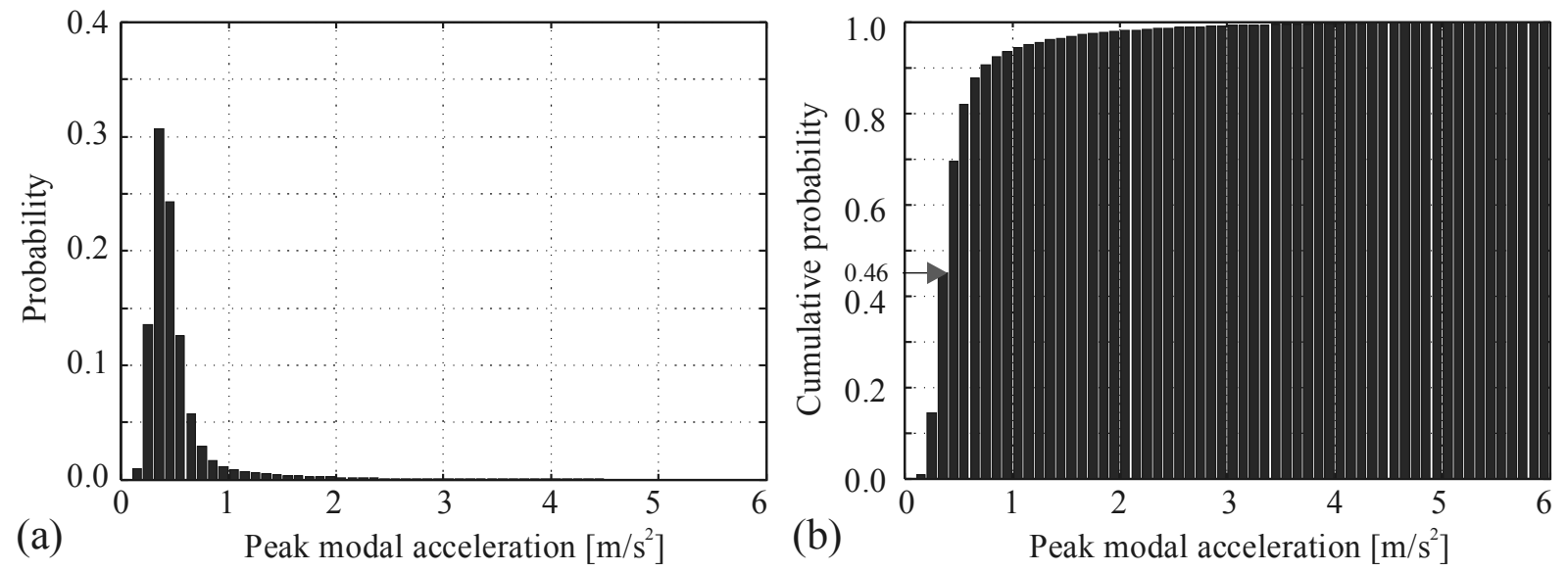

Fig. 10. Footbridge 2 -(a) Probability of certain acceleration level. (b) Cumulative probability that the acceleration level is smaller than or equal to the acceleration level considered.

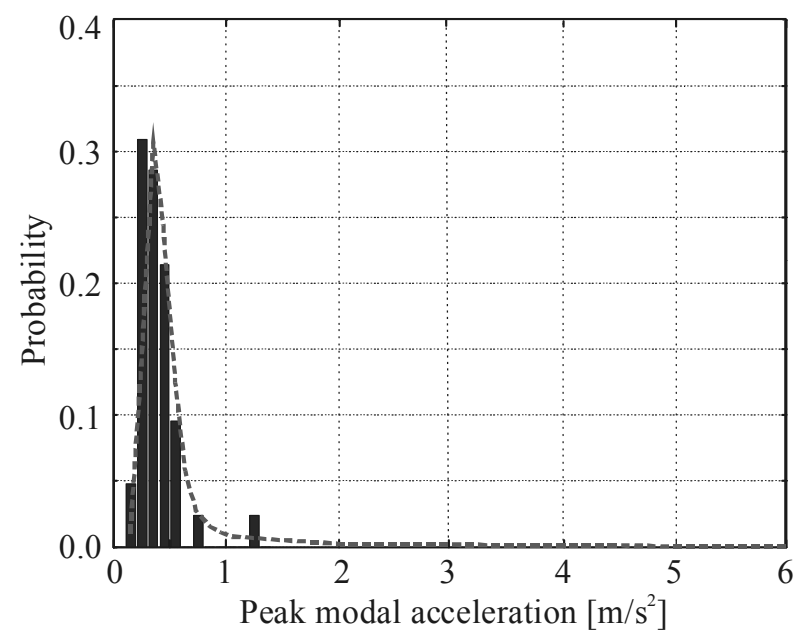

Fig. 11. Histogram of measured peak modal acceleration on Footbridge 2. The calculated values from Figure 10a are presented as dashed line.

Figure 11 shows the probability of different levels of peak modal acceleration measured. It can be seen that the observed probability distribution is very similar to the one calculated in Fig. 10a (and presented by dashed-line in Fig. 11) verifying the probabilistic framework used. A summary of the probabilistic procedure developed is shown in Fig. 12.

It should be noted that the numerical results for Footbridge 2 are quite similar to those acquired experimentally despite the fact that the actual mode shape is not very close to the half-sine function (Fig. 9b). This difference in mode shapes does introduce some errors in the estimation of the peak response. For example, the calculated peak acceleration generated by walking at the resonant frequency at speed of $1.08 \mathrm{~m} / \mathrm{s}$ introduces about $45 \%$ higher peak response than that obtained when the measured shape of the vibration mode was accounted for. Outside the resonance, this difference is smaller being less than $10 \%$ when the walking frequency differs by $5 \%$ or more from the natural frequency of the bridge. The probability that people will walk at or around the resonant frequency of this bridge, which is $1.52 \mathrm{~Hz}$, is small (Fig. 1a), as walking at 1.52 paces per second is quite slow and unnatural pacing rate for most people. This means that the assumption about the half-sine mode shape used in calculations is not expected to have significant influence on the predicted probability distribution of the vibration response. Looking carefully at Fig. 11 which shows the measured and calculated distributions, it could be seen that the maximum values 


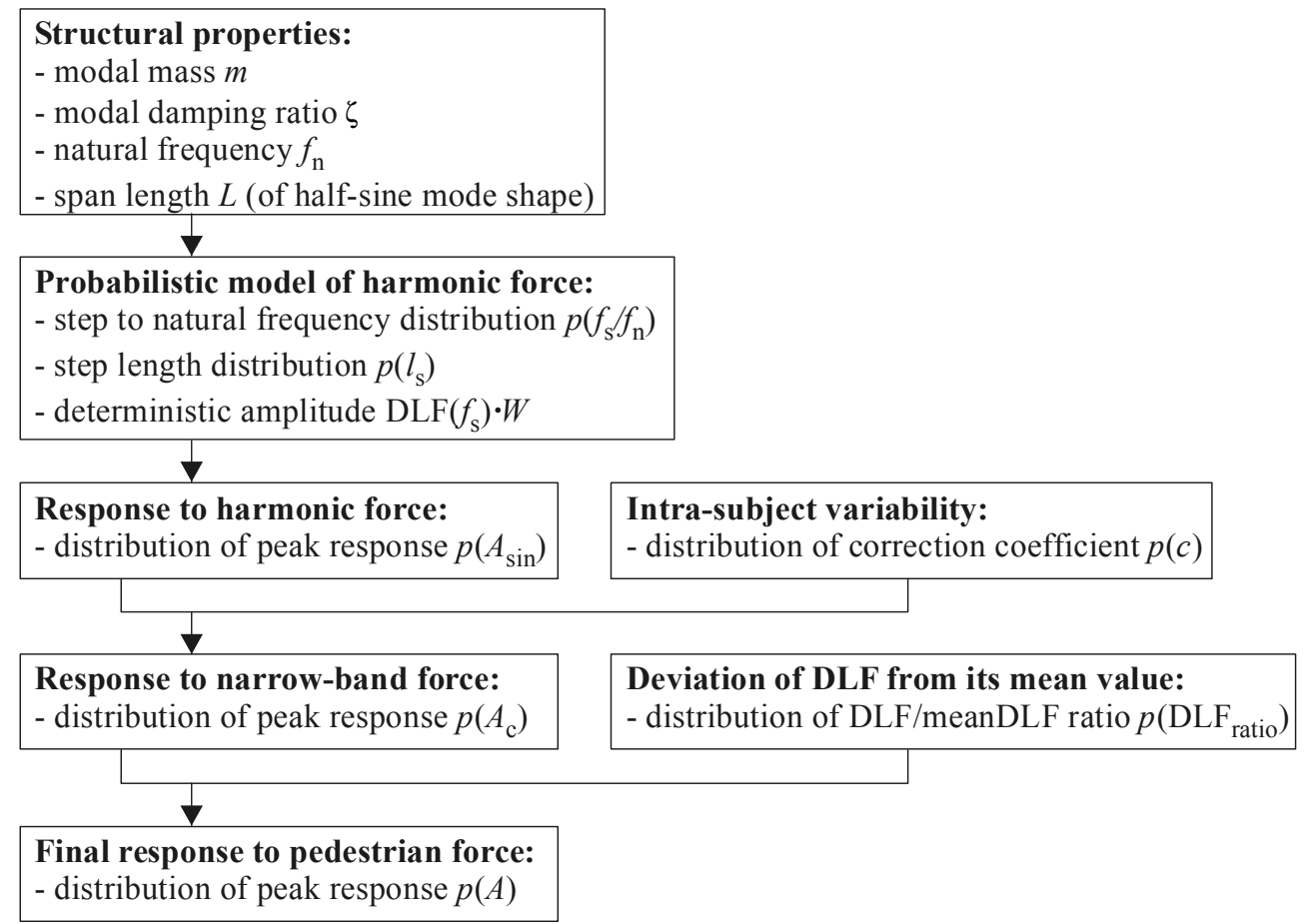

Fig. 12. Summary of the probabilistic calculation procedure.

in the predicted distribution go above $1.3 \mathrm{~m} / \mathrm{s}^{2}$ which was the maximum measured value. However, these high values occur so rarely that they are not significant when interpreting the final results.

\section{Discussion}

In the case of the two footbridges investigated, a probabilistic approach was able to estimate the range of acceleration levels that could be induced by different walkers. As for Footbridge 1, the maximum possible acceleration level was found to be about $0.9 \mathrm{~m} / \mathrm{s}^{2}$, although a negligible percentage of people is capable of generating it. However, it is interesting that during normal multi-pedestrian traffic the peak acceleration level measured was often reaching $0.4 \mathrm{~m} / \mathrm{s}^{2}$ and occasionally went up to $0.6-0.7 \mathrm{~m} / \mathrm{s}^{2}$ [13]. Therefore, on Footbridge 1 only the most efficient human dynamic exciters could induce vibrations that are comparable with the peak acceleration level measured during the normal pedestrian traffic.

On the other hand, for Footbridge 2, majority of pedestrians could induce vibrations up to $1 \mathrm{~m} / \mathrm{s}^{2}$, while the absolute maximum level was around $6 \mathrm{~m} / \mathrm{s}^{2}$. The acceleration range calculated and the shape of its distribution are in very good agreement with the one identified experimentally (Fig. 11). This suggests that the probability distributions used are good descriptor of pedestrian population using the bridge.

It is interesting to compare results of this study with those from the classical deterministic approach. Probably the most often used implementation of this approach is that given in the British Standard BS 5400 [2], which also features in the Canadian design guideline [11]. The walking force therefore is modelled as a resonant sine force. The parameters corresponding to BS 5400 procedure are given in Table 3. The peak modal response is calculated and shown in the last row of the table. It is equal to $0.26 \mathrm{~m} / \mathrm{s}^{2}$ for Footbridge 1 , and $3.12 \mathrm{~m} / \mathrm{s}^{2}$ for Footbridge 2 . Therefore, the "average" person featuring BS5400 generates the acceleration level that is exceeded by only about $7 \%$ and $0.8 \%$ (according to Figs $8 \mathrm{~b}$ and 10b) of test subjects walking across Footbridge 1 and Footbridge 2, respectively. This is because the BS model assumes walking in resonance, which often is not probable walking scenario. Therefore, for the case of two footbridges investigated, the BS model represents a more efficient dynamic exciter than a real- 
Table 3

Parameters for response simulations, according to BS5400 [3]

\begin{tabular}{lll}
\hline Footbridge \# & 1 & 2 \\
\hline Weight $[\mathrm{N}]$ & 700 & 700 \\
Step frequency $[\mathrm{Hz}]$ & 2.04 & 1.52 \\
DLF & 0.257 & 0.257 \\
Walking speed $[\mathrm{m} / \mathrm{s}]$ & 1.84 & 1.37 \\
Peak modal response $\left[\mathrm{m} / \mathrm{s}^{2}\right]$ & 0.26 & 3.12 \\
\hline
\end{tabular}

life average person is. Deterministic models for single pedestrian similar to that of BS5400 feature in numerous footbridge related guidelines, such as ISO 10137 [25], UK National Annex to Eurocode 1 [26] and Eurocode 5 [27]. Therefore, similar obstacles in implementation of these procedures for modelling single pedestrian loading scenario could be expected.

There are schools of thought that justify the BS5400 approach as the one designed to cater for some more complicated (for calculation) load case scenarios, such as normal multi-person pedestrian traffic. However, the peak acceleration value of $0.26 \mathrm{~m} / \mathrm{s}^{2}$ for Footbridge 1 is quite an underestimation of the peak vibration level measured on the same bridge under multi-person pedestrian traffic, being around $0.6-0.7 \mathrm{~m} / \mathrm{s}^{2}[13]$.

As described earlier, the procedure suggested in this paper could be enhanced by introducing probability distribution of people's weight as well as uncertainties in structural dynamic properties in the analysis. This would bring possibility for codification of the probabilistic approach in footbridge design. Given that it is not realistic to expect in depth training of designers in vibration serviceability field, the possible implementation in the design could be realised in at least two ways: either through development of user-friendly software that can then be used in practice, or through development of ready to use charts, similarly to the response spectra developed for series of footbridges by Wan et al. [28].

\section{Conclusions}

A novel probability based framework for predicting vibration response to single person excitation is presented in this paper. The novelty of the approach is characterised by including both the inter- and intra-subject variability in the walking force into the model proposed. The inter-subject variability is included via three probability density functions of forcing amplitude, step frequency and step length while the intra-subject variability is modelled using a probability density function representing (in)ability of people to produce sinusoidal force while walking. This model is applied to two as-built footbridges with known modal properties. In this way a range of acceleration levels that could be generated when a single person is crossing the bridge as well as their probabilities is found. Based on this the cumulative probability that the response will not exceed certain peak acceleration under a single person walking was calculated. This approach to assessing the vibration level under a single person excitation is more informative than the single value that is the outcome of the current design guidelines. The single value from a typical guideline is found neither to be representative of an average walker nor a good estimate of the vibration levels induced by multi-person traffic.

Therefore, the probabilistic procedure developed in this paper draws the attention of the designers to the huge variability in the vibration response that could be induced by different people and presents the way of quantifying it. Additionally, the procedure could be used when designing footbridges that are not very busy and where a single person loading scenario is the most probable.

\section{Acknowledgements}

The authors are grateful to Professor J.M.W. Brownjohn of the University of Sheffield and the National Institute of Education in Singapore for their permission to use the data related to measured walking forces. We also acknowledge the financial support which came from the UK Engineering and Physical Sciences Research Council (EPSRC) for grant reference GR/S14924/01 ("Investigation of the As-Built Vibration Performance of System Built Floors") as well as for grant reference GR/T03000/01 ("Stochastic Approach to Human-Structure Dynamic Interaction”). 


\section{References}

[1] S. Živanović, A. Pavic, P. Reynolds, Vibration serviceability of footbridges under human-induced excitation: a literature review, Journal of Sound and Vibration 279(1-2) (2005), 1-74.

[2] BSI, Steel, concrete and composite bridges. Specification for loads, BS 5400: Part 2, British Standard Institution, London, 1978.

[3] J.M.W. Brownjohn, A. Pavic and P. Omenzetter, A spectral density approach for modelling continuous vertical forces on pedestrian structures due to walking, Canadian Journal of Civil Engineering 31(1) (2004), 65-77.

[4] S. Živanović, A. Pavic and P. Reynolds, Probability-based prediction of multi-mode vibration response to walking excitation, Engineering Structures 29(6) (2007), 942-954.

[5] P. Dallard, A.J. Fitzpatrick, A. Flint, S. Le Bourva, A. Low, R.M. Ridsdill-Smith and M. Willford, The London Millennium Footbridge, The Structural Engineer 79(22) (2001), 17-33.

[6] J.M.W. Brownjohn, P. Fok, M. Roche and P. Omenzetter, Long span steel pedestrian bridge at Singapore Changi Airport - part 2: crowd loading tests and vibration mitigation measures, The Structural Engineer 82(16) (2004), 28-34.

[7] J.H.G. Macdonald, Pedestrian-induced vibrations of the Clifton Suspension Bridge, Proceeding of the Institution of Civil Engineers, Structures and Buildings 161(2) (2008), 69-77.

[8] E. Caetano, A. Cunha and C. Moutinho, Implementation of passive devices for vibration control at Coimbra footbridge, in Proceedings of the 2nd International Conference on Experimental Vibration Analysis for Civil Engineering Structures, Porto, 24-26 October 2007.

[9] S.C. Kerr, Human induced loading on staircases, PhD Thesis, University College London, Mechanical Engineering Department, UK, 1998.

[10] A. Ebrahimpour, A. Hamam, R.L. Sack and W.N. Patten, Measuring and modeling dynamic loads imposed by moving crowds, ASCE Journal of Structural Engineering 122(12) (1996), 1468-1474.

[11] OHBDC, Ontario Highway Bridge Design Code. Highway Engineering Division, Ministry of Transportation and Communication, Ontario, Canada, 1983.

[12] S. Živanović and A. Pavic, Probabilistic Assessment of Human Response to Footbridge Vibration. Journal of Low Frequency Noise, Vibration and Active Control 28(4) (2009), 255-268.

[13] S. Živanović S, A. Pavic, P. Reynolds and P. Vujović, Dynamic analysis of lively footbridge under everyday pedestrian traffic, Sixth European Conference on Structural Dynamics (EURODYN 2005) 1 (2005), 453-459, Paris, France, 4-7 September.

[14] H. Bachmann, A.J. Pretlove and H. Rainer, Vibrations induced by people, in: Vibration Problems in Structures: Practical Guidelines, Birkhäuser Verlag 1995, Basel.

[15] Y. Matsumoto, T. Nishioka, H. Shiojiri and K. Matsuzaki, Dynamic design of footbridges, IABSE Proceedings (No. P-17/78) (1978), 1-15.

[16] S.C. Kerr and N.W.M. Bishop, Human induced loading on flexible staircases, Engineering Structures 23 (2001), $37-45$.

[17] A. Pachi and T. Ji, Frequency and velocity of people walking, The Structural Engineer 83(3) (2005), 46-40.

[18] C. Sahnaci and M. Kasperski, Random loads induced by walking, The Sixth European Conference on Structural Dynamics (EURODYN 2005) 1 (2005), 441-446, Paris, France, 4-7 September.

[19] D.C. Montgomery and G.C. Runger, Applied Statistics and Probability for Engineers, John Wiley \& Sons 1999, New York.

[20] F. Ricciardelli, C. Briatico, E.T. Ingolfsson and C.T. Geogakis, Experimental validation and calibration of pedestrian loading models for footbridges. Proceedings of the 2nd international conference on Experimental Vibration Analysis for Civil Engineering Structures (EVACES'07), Porto, Portugal, 24-26 October 2007.

[21] F.W. Galbraith and M.V. Barton, Ground loading from footsteps, The Journal of The Acoustical Society of America 48(5) (1970), $1288-1292$.

[22] DH. Department of Health 2005, UK, webpage: http://www.dh.gov.uk/PublicationsAndStatistics/PublishedSurvey/HealthSurveyFor England/HealthSurveyResults.

[23] S. Živanović, A. Pavic and P. Reynolds, Human-structure dynamic interaction in footbridges, Proceedings of the Institution of Civil Engineers: Bridge Engineering 158(4) (2005), 165-177.

[24] D.J. Inman, Engineering Vibration, Prentice Hall, Inc., Upper Saddle River, New Jersey, 2001.

[25] ISO 10137:2007, Bases for design of structures - Serviceability of buildings and walkways against vibration. International Organization for standardization.

[26] UK National Annex to Eurocode 1: Actions on Structures - Part 2: Traffic Loads on Bridges, NA to BS EN 1991-2:2003, 2008, British Standards Institution.

[27] EN 1995-2:2004, Eurocode 5: Design of timber structures - Part 2: Bridges, British Standards Institution.

[28] K. Wan, S. Živanović and A. Pavic, Design spectra for single person loading scenario on footbridges. Proceedings of IMAC XXVII, Orlando, Florida, USA, 9-12 February, 2009. 

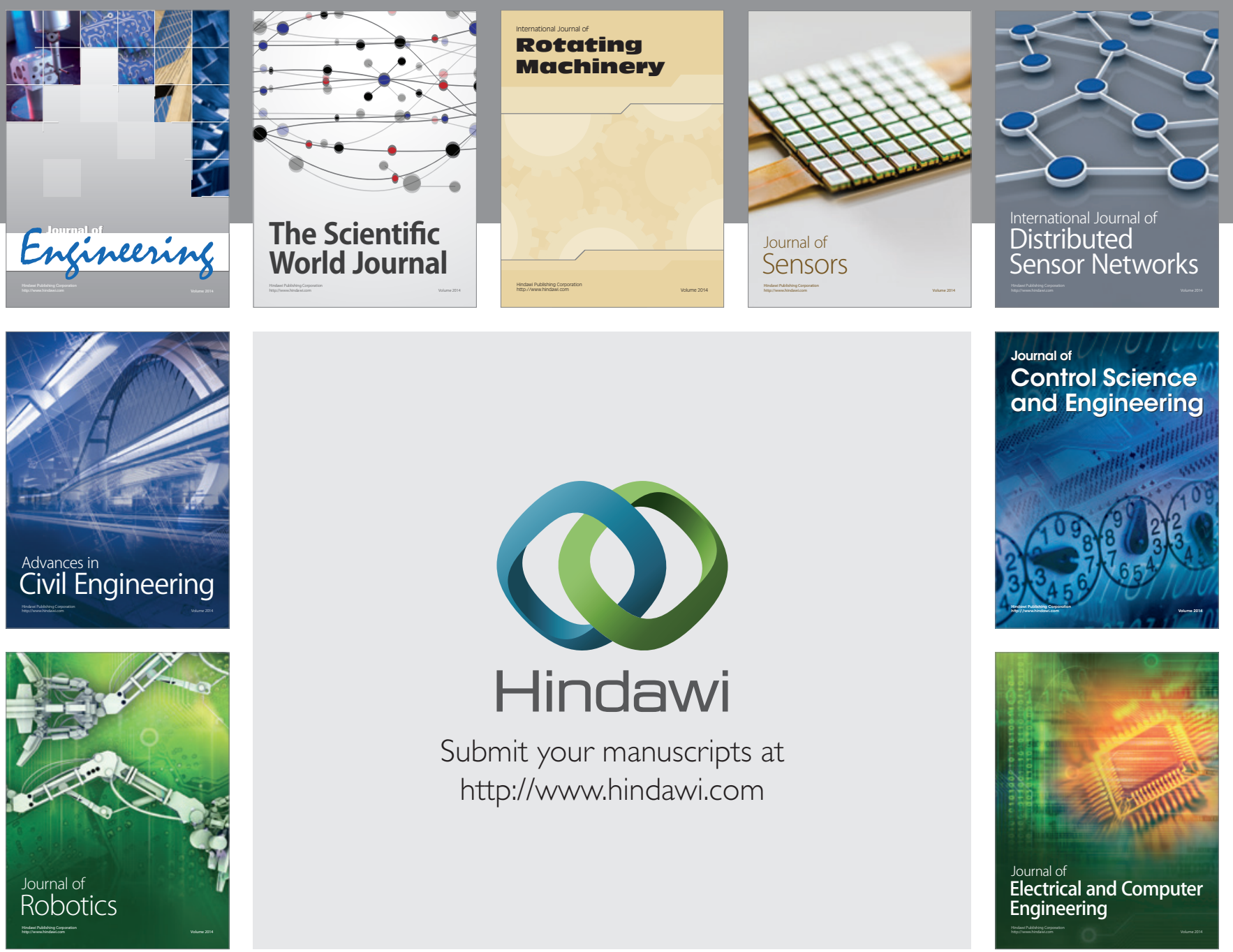

Submit your manuscripts at

http://www.hindawi.com
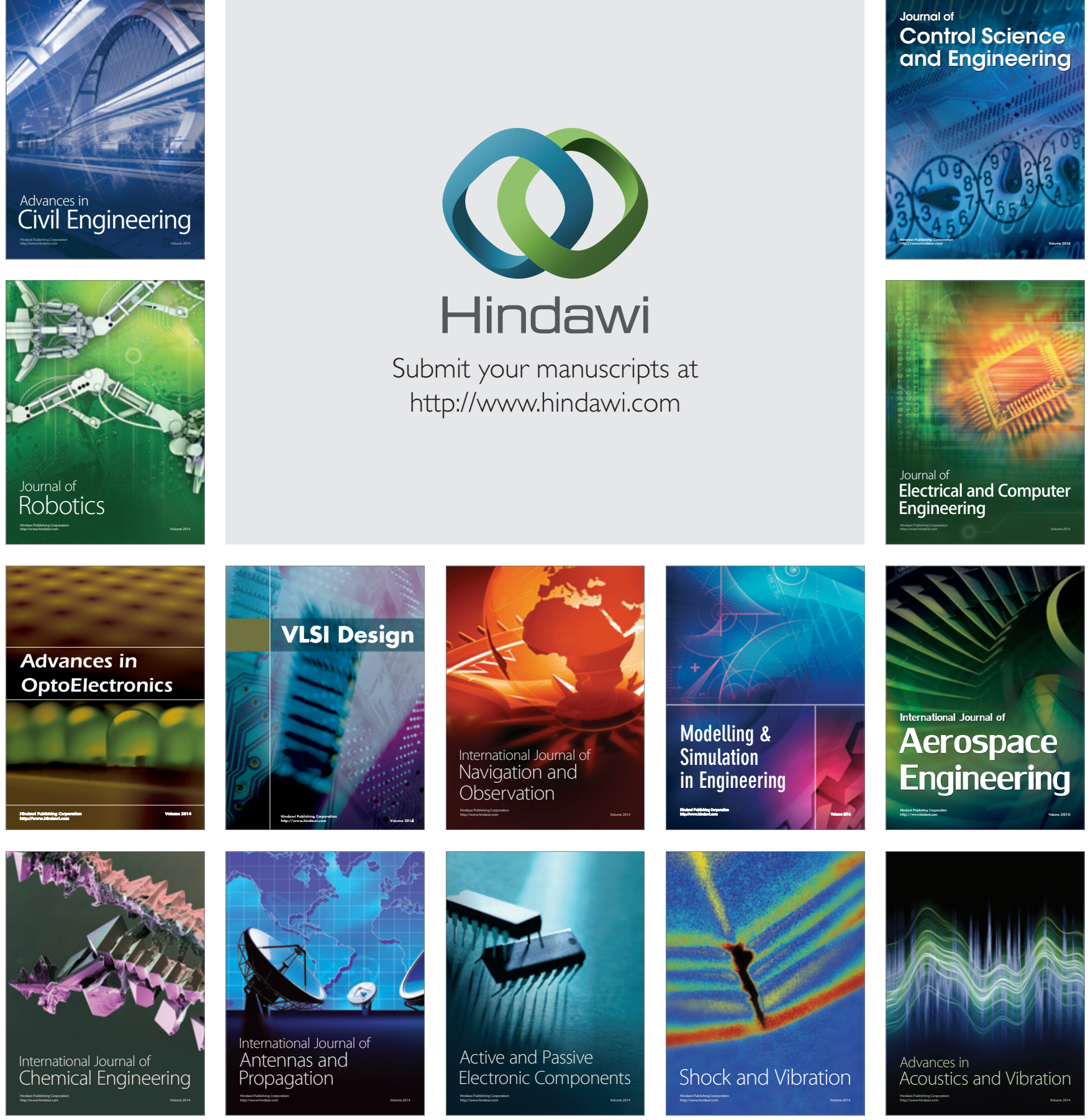